\title{
A three-dimensional model for the high-energy emissions from the Crab pulsar
}

\author{
J.Takata*, H.-K.Chang ${ }^{\dagger}$ and K.S.Cheng ${ }^{* *}$ \\ *ASIAA/National Tsing Hua University-TIARA, Taiwan \\ ${ }^{\dagger}$ Department of Physics and Institute of Astronomy, National Tsing Hua University, Taiwan \\ ${ }^{* *}$ Department of Physics, University of Hong Kong, China
}

\begin{abstract}
We apply a three-dimensional outer gap accelerator model to the Crab pulsar for examining the light curve, phaseresolved spectrum and polarization. The curvature radiation process of the high-energy particles in the gap is calculated with an assumed three-dimensional structure of the accelerating electric field. We calculate the synchrotron self-inverse Compton process from the secondary pairs, which will be the observed emissions in optical to $\gamma$-ray regions. We compute radiation transfer for each radiated beam in three-dimensional geometry.
\end{abstract}

Keywords: Crab pulsar, high-energy emissions, theory

PACS: 90

\section{INTRODUCTION}

After 40 years since the discovery of the pulsar, the high-energy emission mechanism and its site in the pulsar magnetosphere have not been conclusive up to now. In the next decade, the GLAST LAT will measure the phaseresolved spectrum in the range of $10 \mathrm{MeV}$ to $300 \mathrm{GeV}$ with higher sensitivity than the EGRET. These future observations will contribute to diagnose the non-thermal emission physics in the pulsar magnetosphere. The outer gap model is one of major acceleration model of the pulsar magnetosphere. However, there are no works which calculate light curve, the spectrum and the polarization characteristics, simultaneously. To calculate above emission properties, we require a three-dimensional model. Cheng et al. (2000) examined the light curve with the traditional outer gap geometry. Takata et al. (2007) calculated the synchrotron radiation with a recent proposed outer gap geometry and explained the observed polarization data in optical band (Kanbach et al. 2005). In this paper, we further develop the three-dimensional model by computing the radiation transfer of both synchrotron and inverse-Compton processes, and we calculate the phase-resolved spectrum with the light curve and the polarization in optical to $\mathrm{GeV}$ regions.

\section{MODEL}

As indicated by the two-dimensional electrodynamical study (Takata et al. 2006, and reference therein), we assume that the outer gap is extending from near the stellar surface to near the light cylinder, while Cheng, et al. (2000) considered the traditional geometry, in which the gap starts from the null charge surface of the Goldreich-Julian charge density.

For the Crab pulsar, the outer gap is geometrically thin in the trans-field direction compared with the light radius. In such a case, the accelerating electric field is approximately described by $E_{\| \mid}(r)=\Omega B(r) f^{2}(r) R_{l c}^{2} / c s(r)$, where $B(r)$ is the strength of the magnetic field, $s(r)$ is the curvature of the field lines, $R_{l c}$ is the light radius and $f$ is the local gap thickness in units of the light radius. The particles are accelerated immediately up to $\Gamma_{e}(r)=\left[3 s^{2}(r) E_{\|} / 2 e\right]^{1 / 4}$ and emit $\gamma$-ray photons with typical energy of $E_{c}=3 h \Gamma_{e}^{3} c / 4 \pi s(r)$ via the curvature radiation process. For the Crab pulsar, most of the primary photons escaping from the outer gap will convert into the secondary pairs outside the gap by photon-photon pair-creation process with the magnetospheric $X$-ray photons. The secondary pairs emit optical $\mathrm{MeV}$ photons via the synchrotron process and above $\mathrm{MeV}$ photons with the inverse Compton process. We assume that the secondary pairs emit the synchrotron photons in the direction of the motion. We trace the three dimensional path of the synchrotron photons in the magnetosphere and calculate the inverse-Compton process by simulating the scattering points. Equivalently, we compute the equation of the radiation transfer, $d I / d s=j_{s}+j_{i}$, where $j_{s}$ and $j_{i}$ are respectively volume emissivities of the synchrotron and the inverse-Compton processes. The effects of the absorption and scattering off from the line of sight are negligible. We also calculate the Stokes parameter $Q$ and $U$.

CP921, The First GLAST Symposium

edited by S. Ritz, P. Michelson, and C. Meegan

(c) 2007 American Institute of Physics 978-0-7354-0431-1/07/\$23.00 


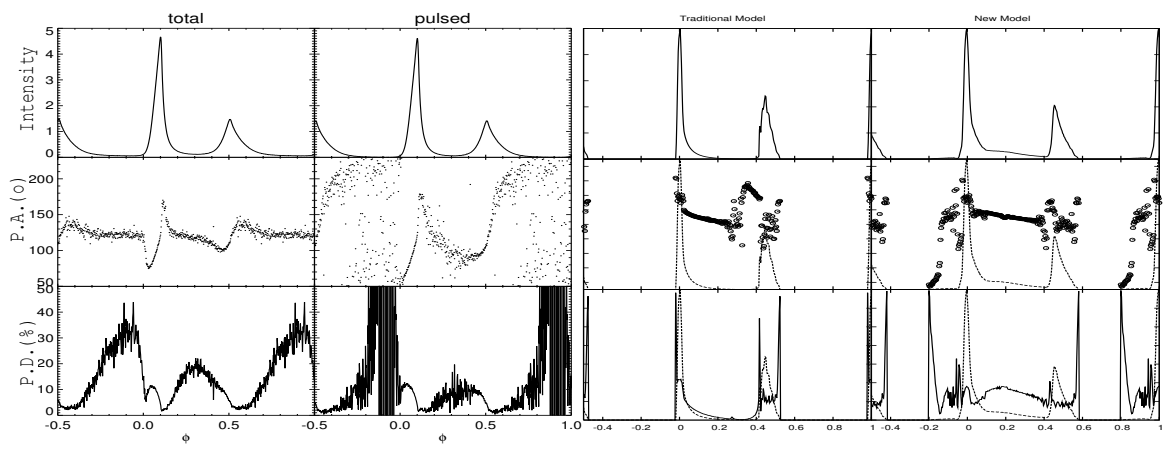

FIGURE 1. Variations of the intensity (upper), polarization position-angle (middle) and degree (lower) as a function of (abscissa). Left two columns show the observed polarization data for the total emissions and the pulsed emissions (after subtracting the DC level). Right two columns show the predicted polarization characteristics at $1 \mathrm{eV}$ for the traditional and the new outer gap geometries. The figures for the Crab optical data was transcribed from Dyks et al. (2004).

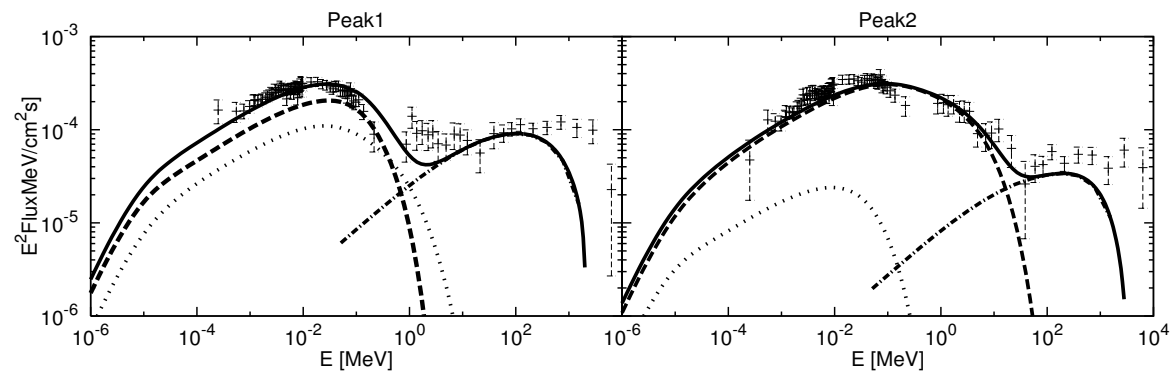

FIGURE 2. Phase-resolved spectra for the first peak the first peak and the second peaks. Data are taken from Kuiper et al. (2000).

\section{RESULTS}

Figure 1 compares the predicted polarization characteristics in the optical band predicted by the traditional and the present outer gap geometries with the data. For data, the figure displays the polarization for the total emissions and the pulsed emissions after subtracting DC level. We find that the traditional outer gap model can not explain variations of the polarization position-angle and degree. On the other hand, the results for the new outer gap geometry are in general consistent with the observed characteristics for the pulsed emissions. Figure 2 shows the phase-resolved spectra of the emissions at Peak1 phase (defined by -0.05 to 0.05 phase in right two columns of the Figure 1) and Peak 2 phase (defined by 0.4 to 0.51 phase). The calculated total emissions (solid line) in the Figure 2 are composed of the synchrotron emissions below (dotted line) and beyond (dashed line) the null charge surface and the inverse-Compton emissions (dashed-dotted line). We find that both the synchrotron components contribute to the total emissions at the Peak1. In the Peak2, the synchrotron emissions beyond the null charge surface dominate in the total emissions. We can see that the predicted phase-resolved spectra are consistent with the observations in wide energy bands. The inverseCompton model does not predicted observed flux in the $\mathrm{GeV}$ regions. $\mathrm{In} \mathrm{GeV}$ regions, we consider that the residual primary curvature photons, which escape the pair-creation process, will contribute to the observed emissions.

\section{REFERENCES}

1. K. S. Cheng, M. Ruderman and L. Zhang, 2000, ApJ, 537, 964

2. J. Dyks, A. K. Harding and B. Rudak, 2004, ApJ, 606, 1125

3. G. Kanbach, A. Słowikoska, A., S. Kellner and H. Steinle, 2005, AIP Conference Proceeding, 801, 306

5. L. Kuiper et al. 2001, A\&A, 378, 918

5. J. Takata, H.-K. Chang and K. S. Cheng, 2007, ApJ, 656, 1044

6. J. Takata, S. Shibata, K. Hirotani and H.-K. Chang, 2006, MNRS, 366, 1310 
Copyright of AIP Conference Proceedings is the property of American Institute of Physics and its content may not be copied or emailed to multiple sites or posted to a listserv without the copyright holder's express written permission. However, users may print, download, or email articles for individual use. 